\title{
Optical Properties of CNT Arrays Growth in Porous Anodic Alumina Templates
}

\author{
Ratnakar Pandu ${ }^{\dot{A}^{*}}$, Ramakrishnudu $^{\dot{\mathrm{A}}}$ and C.V Reddy ${ }^{\dot{\mathrm{A}}}$ \\ ${ }^{\dot{A}}$ Department of Mechanical Engineering, St. Martin's Engineering College, Kompally, Hyderabad India
}

Accepted 10 January 2014, Available online 01 February 2014, Special Issue-2, (February 2014)

\begin{abstract}
The synthesized ordered carbon nanotube (CNT) arrays in porous anodic alumina (PAA) matrix, and have characterized their total optical reflectance and bi-directional reflectance distribution function after each processing step of the microwave plasma chemical vapor deposition process (MPCVD). For a PAA sample without CNT growth, the reflectance shows an oscillating pattern with wavelength that agrees reasonably with a multilayer model. During the MPCVD process, heating the sample significantly reduces the reflectance by 30-40\%, the plasma treatment reduces the reflectance by another 5-10\%, and the CNT growth further reduces the reflectance by 2-3\%. After an atomic layer deposition (ALD) process, the reflectance increases to the embedded CNT arrays. After etching and exposure of CNT tips, the reflectance almost returns to the original pattern with slightly higher reflectance. Bi-directional reflectance distribution function (BRDF) measurements show that the CNT-PAA surface is quite specular as indicated by a large lobe at the specular angle, while the secondary lobe can be attributed to surface roughness.
\end{abstract}

Keywords: Carbon Nanotubes, plasma chemical vapor deposition, porous anodic alumina, CNT arrays

\section{Introduction}

In the year 1991, Iijima first developed carbon nanotubes using an arc-discharge method (Iijima, 1991). Carbon nanotubes (CNTs) are composed of individual sheets of graphene rolled up into cylindrical tubes that have only a few nanometers thick. CNTs are two types: single walled carbon nanotubes (SWCNTs), composed of one graphene sheet, and multi-walled carbon nanotubes (MWCNTs), composed of several sheets wrapped around each other in concentric rings.

CNTs can be grown in arrays for promising optical, electrical, and thermal applications. In general, optical properties of CNT arrays depend on both the dielectric function of each individual CNT and the long-range configuration of the array. To date, many interesting optical properties have been observed in CNT arrays grown without a template, such as photonic crystal effects (K. Kempa et al, 2003 and E. Lidorikis et al, 2009), directional emission (K. Kempa et al, 2007 and X. J. Wang et al, 2009), wavelength-selective emission and polarization-dependent reflection (S. Shoji, et al, 2008), and high absorptivity (Z. P. Yang et al, 2008 and H. Bao et al, 2010). On the other hand, CNT arrays can also be grown in templates, among which porous anodic alumina (PAA) has been well-studied (F. Keller et al, 1953). The configuration of CNT arrays grown in PAA holds promise for electronic devices based on carbon nanotubes (M. R. Maschmann et al, 2006 and A. D. Franklin et al, 2008). PAA is an arrangement of semi-ordered pores that are the

*Corresponding author: Ratnakar Pandu

DOI: http://dx.doi.org/10.14741/ijcet/spl.2.2014.60 result of anodization on an $\mathrm{Al}$ film, and the pores protect CNTs grown in the microwave plasma chemical vapor deposition (MPCVD) system. The presence of CNTs can possibly modify the optical properties of the PAA or exhibit optical antenna effect. Such knowledge is also useful for using CNT arrays as additives to tune the optical properties of the matrix material. However, no measurements or analysis of optical properties have been performed on template-grown CNT arrays so far. In this work, we have synthesized CNT arrays in PAA templates and measured their optical properties. Well-ordered and high-yield CNT growth in porous anodic alumina (PAA) templates was established by varying conditions such as electrical bias and plasma treatments. Controlled variation of process (ALD, etching) has been achieved. Optical reflectance has been characterized on CNT arrays samples, and tunable thermal radiative properties in total reflectance and spectra have been observed.

\section{Growth and preparation of CNT arrays}

Some of the earliest studies of nanoscale templating involved porous anodic alumina (PAA). PAA is formed by anodic oxidation of $\mathrm{Al}$ in an acidic electrolyte. The $\mathrm{Al}$ is typically either a foil that is several tens of microns thick or a substrate-supported thin film. The resulting alumina template consists of vertical pores in a naturally occurring hexagonal arrangement. The pore diameter is linearly dependent on the anodization electrolyte and voltage demonstrated pore diameters range from sub-10 $\mathrm{nm}$ to several hundred nanometers. Today PAA is used in myriad applications, from a dye-support on the casing of

325 | International Conference on Advances in Mechanical Sciences 2014 
commercial aluminum products to a template for supporting the electrochemical formation of nanofibres (A. P. Li et al, 1998 and E. C. Walter et al, 2002). The earliest reports of CNTs templated in PAA involve MWCNTs synthesized with or without deposited catalyst in the pores. When synthesizing MWCNTs in PAA without a catalyst, the main formation mechanism is the adsorption of carbon on the alumina pore walls by the decomposition of hydrocarbons, and this process yields tubes of low crystalline quality (S. H. Jeong et al, 2004 and H. Y. Jung et al, 2005). MWCNT synthesis methods utilizing a catalyst include the use of $\mathrm{Co}$ or $\mathrm{Ni}$ deposited within the pores followed by MWCNT synthesis. These processes yield MWCNTs that take on the diameter of the pores and typically have a high concentration of disordered carbon, but they provide excellent control of the length and diameter of the MWCNTs by varying the dimensions of the PAA.

However, SWCNT synthesis from customized templates or catalysts is more difficult because of their relatively high activation energy in comparison to MWCNTs (M. R. Maschmann et al, 2007 and S. Hofmann et al, 2005). Additionally, nucleation of SWCNTs requires catalyst particles of diameters less than a few nanometers, a size that is not easily achieved when fashioning a catalyst within a template. These two obstacles of high activation energy and difficult catalyst particle size-control are overcome in the present work by burying a Fe catalyst layer in a PAA template and synthesizing in a hightemperature microwave plasma chemical vapor deposition (MPCVD) environment (S. Hofmann et al, 2005). The plasma helps to lower the required thermal activation energy by providing a separate means for dissociating the hydrocarbon precursors, thus relieving part of the energy burden from the catalyst particle. Secondly, covering Fe with a thin layer (few nanometers) of alumina (as is the case on the pore sidewalls of the modified PAA template) raises the surface diffusion barrier of the catalytic $\mathrm{Fe}$ particles and thus mitigates agglomeration (G. F. Zhong et al, 2005). The catalytically active PAA template is formed by first depositing a 100nm Ti/100nm Al/1 nm Fe/1000 $\mathrm{nm} \mathrm{Al}$ film stack on a $\mathrm{SiO}_{2}$ covered $\mathrm{Si}$ wafer, as shown in Fig. 1(a). The Ti serves as an adhesion layer for the film and a conductive layer for anodization and other post processing. Typically, the first (bottom) Al layer is $100-$ $200 \mathrm{~nm}$ thick, while the top $\mathrm{Al}$ is $200-1000 \mathrm{~nm}$ thick, and the sandwiched Fe layer is approximately $1 \mathrm{~nm}$ thick. Anodization is carried out in an acidic electrolyte maintained at $5^{\circ} \mathrm{C}$ under a constant voltage that is applied with respect to a Pt gauze counter electrode. While several electrolytes can be used in the formation of PAA, $0.3 \mathrm{M}$ oxalic acid provides pores of $\approx 20 \mathrm{~nm}$ diameter at an anodization voltage of $40 \mathrm{~V}$, which is a good average size when working with the present catalytically active PAA. Larger pores or smaller pores can be obtained by anodizing in phosphoric acid or sulfuric acid, respectively (S. Fournier-Bidoz et al, 2004).

Anodization of $\mathrm{Al}$ occurs by the exchange of $\mathrm{Al} 3+$ anions from the $\mathrm{Al}$ film with $\mathrm{O} 2-$ and $\mathrm{OH}$ cat-ions from the electrolyte. The exchange of these ions results in two main reactions: 1) the growth of $\mathrm{Al} 2 \mathrm{O} 3$ at the $\mathrm{Al} / \mathrm{Al} 2 \mathrm{O} 3$ interface and 2) the dissolution of $\mathrm{Al}_{2} \mathrm{O}_{3}$ at the $\mathrm{Al}_{2} \mathrm{O}_{3}$ /electrolyte interface (O. Jessensky et al, 1998). These reactions cause the formation of alumina pores that proceed perpendicularly from the surface of the Al film. Upon reaching the embedded Fe layer, the anodization current rises by approximately $20 \mathrm{~mA}$ as the growth-front of the PAA breaks through the thin Fe layer and continues to grow into the bottom $\mathrm{Al}$ layer. In this way, the $\mathrm{Fe}$ catalyst becomes embedded in the $\mathrm{Al}_{2} \mathrm{O}_{3}$ film, exposing it for catalytic activity at the inner pore sidewalls. Anodization is complete when the current falls below $1 \mathrm{~mA}$, indicating complete oxidation of the $\mathrm{Al}$ and termination of the $\mathrm{Al}_{2} \mathrm{O}_{3}$ formation at the Ti layer.
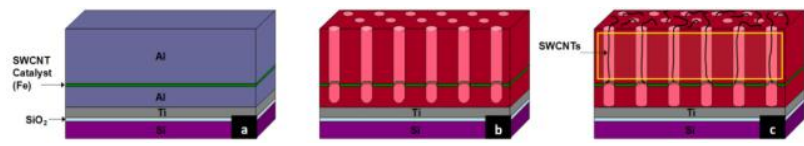

Fig.1. Cross-sectional schematic of: (a) metal film stack deposited on Si wafer, (b) PAA film after anodization, and (c) SWCNTs emerging from PAA pores after undergoing MPCVD synthesis; the box highlights the vertical SWCNT channels.

Synthesis of the SWCNTs from the catalytically active PAA is accomplished in a hydrogen-fed MPCVD reactor with methane as the hydrocarbon precursor gas. Observations indicate that only one SWCNT nucleates in any given pore, likely because of the limited gaseouscarbon supply and the relatively high activation energy required for nucleation. After nucleating at the sidewall of a pore, the SWCNT proceeds to grow vertically to the surface of the PAA, toward the carbon supply. Evidence of this vertical growth is apparent in cross-sectional fieldemission scanning electron microscope (SEM) images showing freestanding vertical SWCNTs, as shown in Fig. 2(a). However, most SWCNTs grow too long to remain vertical and instead lay horizontally across the PAA surface as seen in Fig. 2(b).
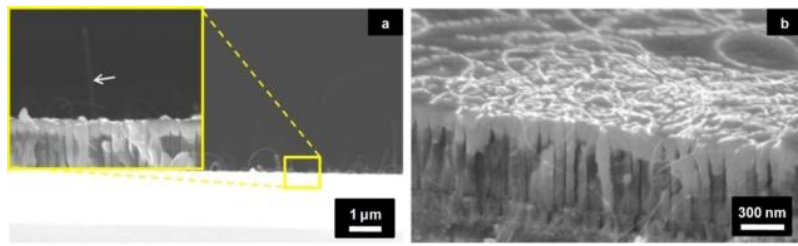

Fig.2. Cross-sectional SEM images of (a) SWCNTs growing vertically from PAA and (b) SWCNTs draping along the PAA surface and appearing as bright strands.

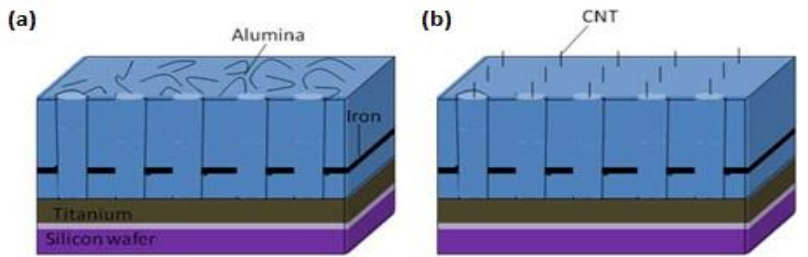

Fig.3. (a) CNTs in PAA sample after ALD deposition of alumina; (b) CNTs in PAA sample after ALD deposition and final etches. 

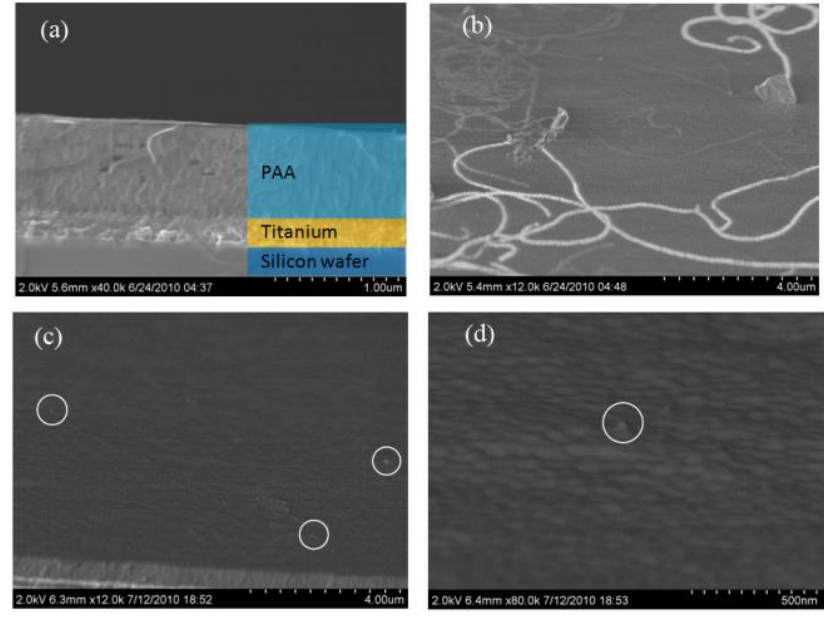

Fig.4. (a) PAA sample with CNT growth and alumina coating from an ALD process, with a transparent color overlay on the right side to indicate the different parts of the metal stack. The iron layer was coated by the alumina. (b) Top view of PAA sample with CNT growth and alumina coating from an ALD process. (c) An RIE etch to expose CNT tips, and some examples of tips are circled. (d) Zoomed in view of a CNT tip on the surface of the sample after anodization, CNT growth, ALD process of alumina, and final etches to obtain CNT tips. The tip is circled.

After CNT growth, the sample was coated with $30 \mathrm{~nm}$ of alumina by atomic layer deposition (ALD). The alumina conformally coats the sample; consequently the film is uniform in thickness and fills the pores while simultaneously coating the top surface. This results in an alumina sample $30 \mathrm{~nm}$ thicker than before with the pores filled. The CNTs remain on the surface, but they are coated with the alumina, as seen in Fig. 3(a). After the ALD process, the film is etched away using chromic acid for 6 minutes. Most of the CNTs are then etched away using an RIE process of $60 \mathrm{sccm}$ of argon at $1 \mathrm{mtorr}$ for 30 seconds at $300 \mathrm{~W}$ of coil power, leaving just tips sticking out of the sample. This results in an alumina sample of the same thickness as the previous PAA with CNTs sample, only with filled pores and CNT tips, to be used in measurements that call for vertical CNTs as in Fig. 3(b). A labeled microscope cross-sectional image of PAA with CNT growth and ALD coating is shown in Fig. 4(a), with a top view of the same sample in Fig. 4(b). A top view of the sample after growth, ALD coating, and final RIE etch is shown in Fig. 4(c), with a magnified view of a CNT tip in Fig. 4(d).

\section{Characterization of optical properties of ordered CNT arrays}

\subsection{Hemispherical reflectance of CNT arrays grown in PAA}

The hemispherical reflectance of the CNT array samples has been measured by a PerkinElmer Lambda 950 spectrometer with an integrating sphere. The experimental setup is shown in Fig. 5. The integrating sphere reflectance measurement allows for data to be taken over a wavelength range from $250 \mathrm{~nm}$ to $1100 \mathrm{~nm}$.

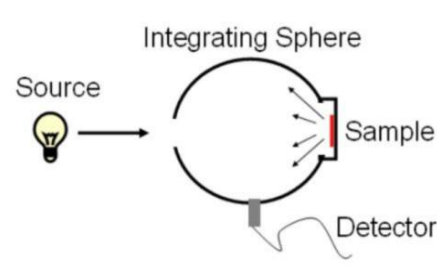

(a)

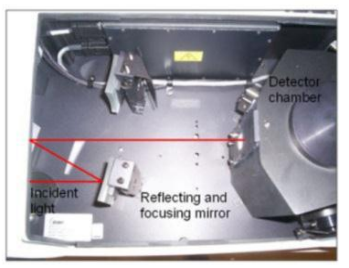

(b)
Fig.5 (a) A sketche of integrating sphere measurement. (b) The actual experimental set-up.

The hemispherical reflectance for the PAA sample without CNT growth is shown in Fig.6, along with the modeling results. Our model considers a three-layer system where a PAA layer with a thickness of d of $750 \mathrm{~nm}$ is sandwiched between a semi-infinite air layer and a semi-infinite titanium layer as the substrate. Each layer is assigned a complex refractive index $\mathrm{m}=\mathrm{n}+\mathrm{i}$, where $\mathrm{n}$ is the refractive index, and $\kappa$ is the extinction index, both of which depend on wavelength. For the PAA layer, the $n$ and $\kappa$ values for alumina, adopted from Ref (E. F. Schubert, 2004), are used. Using refractive index and extinction coefficient data of $\mathrm{Ti}$ in Ref (E. F. Schubert, 2004), the penetration depth is found to range from 16.3 to $26.4 \mathrm{~nm}$ for an incidence wavelength range $247-1127$ $\mathrm{nm}$. Hence, the 100-nm Ti layer is much thicker than the penetration depth and can be assumed to be semi-infinite.

Light is incident from air with an angle $\theta 1$ of $8^{\circ}$, which is the closest angle to the normal incidence that can be obtained practically with our spectrometer. Using the reflectance theory of multilayer thin films (J. A. Kong, 1990), the optical reflectance of the PAA layer can be calculated.

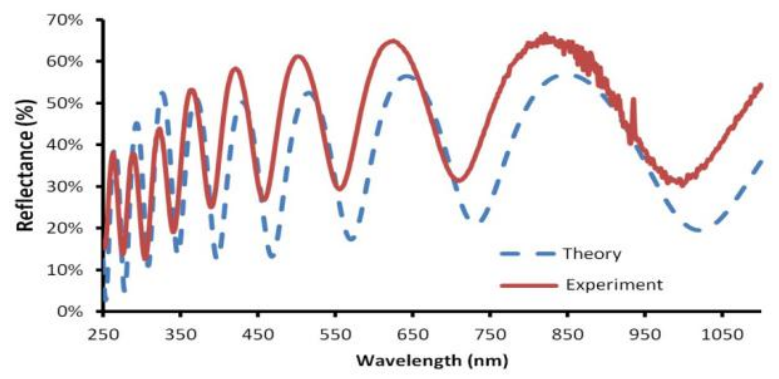

Fig.6 A comparison between the theoretically calculated reflectance at 8 degrees and the measured reflectance for a $750 \mathrm{~nm}$ thick PAA sample.

The experimental and theoretical reflectance profiles show reasonably good agreement, with some discrepancy, as seen in Fig. 6. The oscillating profile manifests the interference of reflected EM fields from the first and second interfaces. This is commonly seen for thin films. The peaks and valleys of the two profiles are similar in periodicity, despite a systematic shift. The model over predicts reflectance between 250 and $350 \mathrm{~nm}$, and underpredicts it from 350 to $1100 \mathrm{~nm}$. Several factors can be responsible for the discrepancy. First, there is probably 
uncertainty in our measurements of the PAA film thickness, so that the actual thickness may not exactly be $750 \mathrm{~nm}$. Second, the anodized holes in actual samples are neglected in the theoretical calculations. Third, the interfaces in the actual samples are probably not ideally smooth, which can make the reflection partially specular and partially diffuse. This will be shown to be the actual case in the next section. The diffuse component will not show any interference effect. In addition, a small uncertainty may exist in the incident angle. We also investigated how different steps in the PECVD process can affect the reflectance of the samples. Heating the sample to $900^{\circ} \mathrm{C}$, introducing the hydrogen plasma, and introducing the methane all change the reflectance properties of the PAA sample. As observed in Fig. 7, when the sample is heated to $900{ }^{\circ} \mathrm{C}$, the reflectance of the sample decreases by $30-40 \%$ Heating the sample to that temperature will cause the metal to expand, cracking and breaking the surface, drastically altering the surface structure and making it rougher, which can enhance light trapping and reduce reflectance. After plasma treatment, the hemispherical reflectance reduces another 5-10 percent, and the CNT growth reduces it further by $2-3 \%$. Adding in the plasma can break the nanopores on the surface, making it rougher. After CNT growth, CNTs add absorption to the light, thus reducing the reflection from the PAA/Ti interface. Overall, the reflectance is reduced more in the near-infrared (NIR) band than in the ultraviolet (UV) band, due to the fact that the samples are already more absorptive in the UV band.

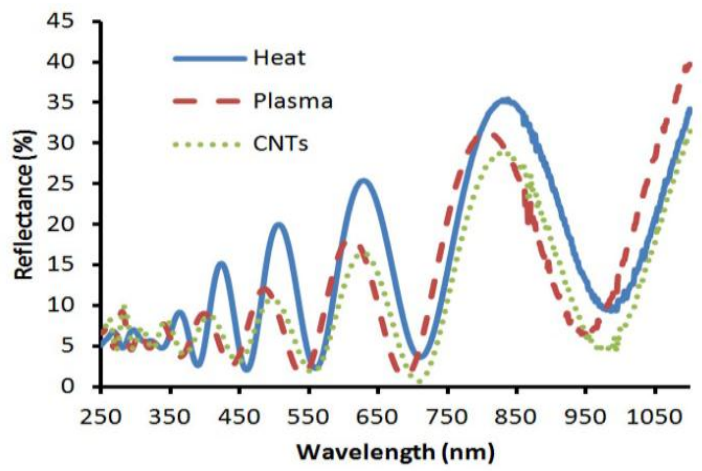

Fig.7 Reflectance measurements at 8 degrees of the three pieces of PAA broken from the same original anodized piece and allowed to complete different steps of the CVD process: heating, plasma treatment, and CNT growth.

We also measured the hemispherical reflectance after each step in the full process: after the PAA construction, after CNT growth, after the atomic layer deposition (ALD) process, and after the final etch to expose the CNT tips. The results for reflectance are shown in Fig. 8. The reflectance profiles for PAA only and after CNT growth have been discussed earlier, and here we focus on the effects of ALD and final etching. The ALD process deposits $30 \mathrm{~nm}$ thick alumina to the top of the device, filling the pores and rendering a relatively smooth surface. Therefore, the reflectance increases and the peaks and valleys shift. After the chromic etch and RIE, however, the reflectance returns almost to the original profile with a slight increase. This is probably due to the exposure of the pores and CNTs.

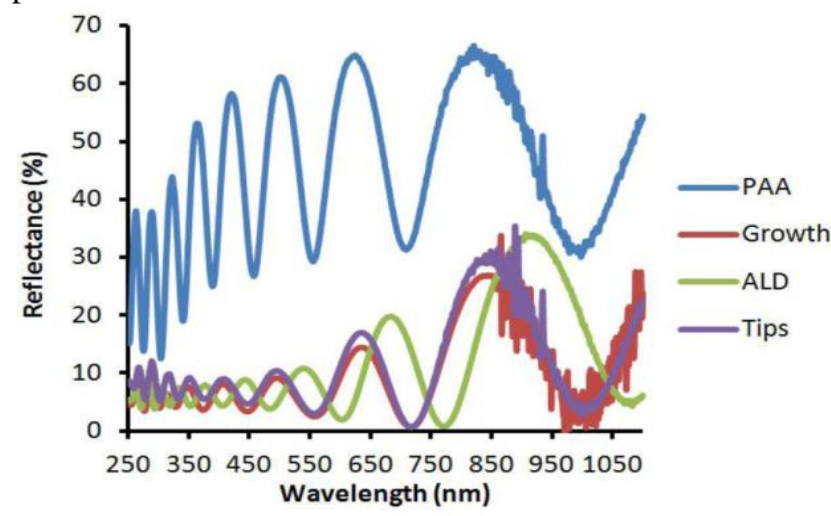

Fig.8. A comparison of total reflectance at 8 degrees after each of the four steps in the full process. PAA: after PAA construction. Growth: after CNT growth. ALD: after the ALD process. Tips: after etching to expose the CNT tips.

\subsection{Bi-directional reflectance distribution function}

To reveal more specifics of the reflectance properties of the samples, we also performed bidirectional reflectance distribution function (BRDF) measurements. In BRDF measurements, the incident angle can be fixed, while the detection angle can vary continuously, allowing for measurements of both specular and diffuse components of scattering. This allows for more specific analysis of samples and angles. Distinct input angles can be chosen for the light, as can exact angles for the detected reflectance. However, if detection angle is set approximately equal to the input angle, specular reflectance dominates the peaks. Normalizing the data allows for more accurate measurements for the reflectance calculations. As shown in Fig. 9, all samples exhibit a large spike in the reflectance distribution function when the detection angle is the same as the incident angle, which indicates that specular scattering is strong. Also, as the incident angle increases, the magnitude of the reflectance peak increases, which is consistent with the trend for most materials? Interestingly, a second lobe exists at a slightly larger angle to the specular peak for all samples. The result with CNTs at $35^{\circ}$ incidence is shown as a clear pattern. The surface roughness of the PAA sample is a major cause of typical observation of strong off-specular peaks due to masking and shadowing of the surface.

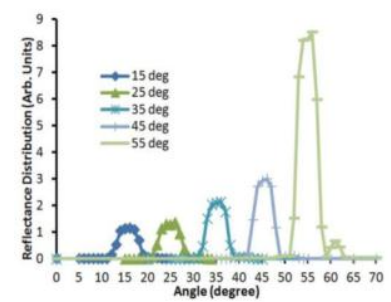

(a)

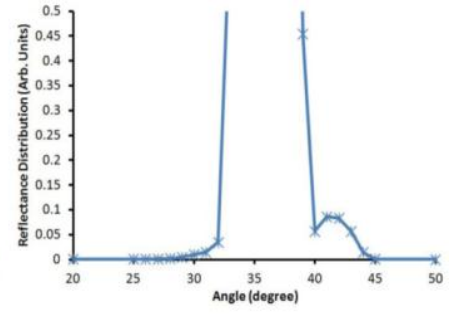

(b)
Fig.9. (a) BRDF measurements of the sample with CNT tips poking out of an alumina substrate as a function of angle. The legend indicates the angle of incidence. (b) A magnified region of the $\mathrm{BRDF}$ at $35^{\circ}$ incidence angle to highlight the secondary lobe. 
The roughness of the pre-anodized aluminum surface has been measured in prior work to be approximately $40 \mathrm{~nm}$ on the preanodized aluminum, and this value is expected to be representative of the porous alumina matrix. Torrance et al. (K. E. Torrance et al and 1967 and A. D. Franklin et al, 2009) described an accurate model for the off-resonance peak in the reflectance from rough surfaces where the roughness is larger than the wavelength. This explanation was found to be valid also for certain subwavelength rough surfaces.

\section{Conclusions}

In summary, ordered CNT arrays were synthesized in PAA matrix under different processing conditions, and characterized their total hemispherical optical reflectance and bi-directional reflectance distribution function (BRDF). For a PAA sample without CNT growth, the reflectance showed an oscillating pattern that can be explained by our model. Heating the PAA spectrally reduces the reflectance by $30-40 \%$, and the plasma treatment reduces the reflectance by another $5-10 \%$. After CNT growth, the reflectance further reduces by $2-3 \%$ since CNT has a high absorption of light. After the ALD process, the reflectance significantly increases due to the embedment of CNT arrays and nanopores. After etching and exposure of CNT tips, the reflectance almost returns to the original pattern with slightly higher reflectance. The bi-directional reflectance distribution function (BRDF) measurements indicate that our CNT-PAA surface is primarily specular, while the secondary lobe can be attributed to surface roughness.

\section{References}

S. Iijima, (1991), Helical microtubules of graphitic carbon, Nature 354(6348), 56-58.

K. Kempa, et al, (2003), Photonic crystals based on periodic arrays of aligned carbon nanotubes, Nano Lett. 3(1), 13-18.E. Lidorikis et al, (2009), Photonics with multiwall carbon nanotube arrays, ACS Nano 3(5), 1238-1248.

K. Kempa et al, (2007), Carbon nanotubes as optical antennae, Adv. Mater. 19(3), 421-426.

X. J. Wang et al (2009), Visible and near-infrared radiative properties of vertically aligned multi-walled carbon nanotubes, Nanotechnology 20(21), 215704.

S. Shoji et al, (2008), Optical polarizer made of uniaxially aligned short single-wall carbon nanotubes embedded in a polymer film, Phys. Rev. B 77(15), 153407.
Z. P. Yang et al, (2008), Experimental observation of an extremely dark material made by a low-density nanotube array, Nano Lett. 8(2), 446-451.

H. Bao et al, (2010), Optical properties of ordered vertical arrays of multi-walled carbon nanotubes from FDTD simulations, Opt. Express 18(6), 6347-6359.

F. Keller et al, (1953) Structural features of oxide coatings on aluminium, J. Electrochem. Soc. 100(9), 411-419.

M. R. Maschmann et al, (2006), Lithography-free in situ Pd contacts to templated single-walled carbon nanotubes, Nano Lett. 6(12), 2712-2717.

A. D. Franklin et al (2008), Independently addressable fields of porous anodic alumina embedded in $\mathrm{SiO} 2$ on $\mathrm{Si}$, Appl. Phys. Lett. 92(1), 013122.

A. P. Li, (1998), Hexagonal pore arrays with a 50-420 nm interpore distance formed by self-organization in anodic alumina, J. Appl. Phys. 84(11), 6023-6026.

E. C. Walter et al, (2002), Electronic devices from electrodeposited metal nanowires, Microelectron. Eng. 61-62, 555-561.

S. H. Jeong et al, (2004), Carbon nanotubes based on anodic aluminum oxide nano-template, Carbon 42(10), 2073-2080.

H. Y. Jung, (2005), Well-ordered semiconducting linearly joined carbon nanotube devices at room temperature, Chem. Phys. Lett. 402(4-6), 535-538.

M. R. Maschmann, (2007), Optimization of carbon nanotube synthesis from porous anodic Al-Fe-Al templates, Carbon 45(11), 2290-2296.

S. Hofmann et al, (2005), Surface diffusion: The low activation energy path for Nanotube growth, Phys. Rev. Lett. 95(3), 036101.

M. R. Maschmann et al, (2006), Vertical single- and doublewalled carbon nanotubes grown from modified porous anodic alumina templates, Nanotechnology 17(15), 3925-3929.

G. F. Zhong et al, (2005),Very high yield growth of vertically aligned single-walled carbon nanotubes by point-arc microwave plasma CVD, Chemical Vapor Deposition 11(3), $127-130$

S. Fournier-Bidoz et al,(2004), Highly ordered nanosphere imprinted nanochannel alumina (NINA), Adv. Mater. 16(2324), 2193-2196

O. Jessensky et al, (1998) Self-organized formation of hexagonal pore arrays in anodic alumina, Appl. Phys. Lett. 72(10), 1173 1175

E. F. Schubert, (2004), Refractive index and extinction coefficient of materials,

J. A. Kong, (1990), Electromagnetic Wave Theory (Wiley)K. E. Torrance et al, (1967), Theory for Off-Specular Reflection from Roughened Surfaces, J. Opt. Soc.Am. 57(9), 1105-1114

A. D. Franklin, (2009), Vertical Carbon Nanotube Devices With Nanoscale Lengths Controlled Without Lithography, IEEE Trans. NanoTechnol. 8(4), 469-476. 